Meta

Journal des tradlucteurs

Translators' Journal

\title{
Étude terminologique des scies manuelles (II)
}

\section{Marie Proulx}

Volume 28, numéro 4, décembre 1983

URI : https://id.erudit.org/iderudit/003871ar

DOI : https://doi.org/10.7202/003871ar

Aller au sommaire du numéro

Éditeur(s)

Les Presses de l'Université de Montréal

ISSN

0026-0452 (imprimé)

1492-1421 (numérique)

Découvrir la revue

Citer cet article

Proulx, M. (1983). Étude terminologique des scies manuelles (II). Meta, 28(4),

358-365. https://doi.org/10.7202/003871ar d'utilisation que vous pouvez consulter en ligne.

https://apropos.erudit.org/fr/usagers/politique-dutilisation/ 


\section{ÉTUDES \\ TERMINOLOGIQUES \\ ET LINGUISTIQUES}

\section{ÉTUDE TERMINOLOGIQUE DES SCIES MANUELLES (II)}

4. NARROW BLADED FRAMED PIT SAW (I846), PIT SAW 2, PIT-SAW 2 : RAQUETTE $_{1}$, SCIE RAQUETTE ${ }_{1}$, SCIE VIOLON (inusité).

Scie à monture de bois, de très grande dimension, à lame fixe, réservée au sciage légèrement cintré dans le sens des fibres du bois.

La raquette est une grande scie montée (env. $2 \mathrm{~m}$ ) de structure identique à la scie de long; elle ne se distingue de celle-ci que par sa lame qui est plus étroite (env. $2,5 \mathrm{~cm}$ à une extrémité et $6 \mathrm{~cm}$ à l'autre); la scie tourne ainsi plus facilement et permet le débit de pièces légèrement cintrées (poutres de navires, jantes de très grandes roues, brancards de charrettes, etc.).

Remarque. "Narrow bladed framed pit saw » est la forme étoffée ; "pit saw " est le terme le plus fréquent.

\section{CONTINENTAL FRAME $S A W_{2}$ : GRANDE SCIE DE TRAVERS, SCIE DE TRAVERS ${ }_{1}$.}

Scie à monture de bois, de grande dimension, à lame fixe, réservée au SCIAGE CINTRÉ (curved sawing; compass sawing) de débit second à travers les fibres du bois.

La grande scie de travers fait partie de l'outillage commun aux artisans du bois. C'est une scie montée de grande dimension $(100$ à $150 \mathrm{~cm})$. La lame est large $(8 \mathrm{à} 10 \mathrm{~cm}$ ), épaisse (env. 1,2 cm), et FIXE (fixed blade) dans le plan de la monture; la DENTURE est TRIANGULAIRE ISOCÈLE, (peg teeth; pegged teeth; fleam teeth), ce qui permet à la scie d'agir dans les deux sens de son mouvement de va-et-vient. Cette scie est MANOeUvRÉE PAR DeUX hOMMEs (two-man saw), et est utilisée pour débiter les BoIs courbes (compass wood)) (limons, p. ex.) et pour exécuter les courbes en travers des fibres du bois. La pièce à couper est posée sur des tréteaux.

\section{CONTINENTAL FRAME SAW 3 : SCIE DE CHARPENTIER, SCIE DE TAILLE DU CHARPENTIER.}

Scie à monture de bois, de grande dimension, à lame fixe, réservée au sciage cintré de débit second. 
La scie de charpentier est presque identique à la grande scie de travers; c'est une scie de grande dimension (1 à $1,5 \mathrm{~m})$ dont la lame est large $(8 \mathrm{à} 10 \mathrm{~cm})$ et fixe dans le plan de la monture. Cependant, l'épaisseur de sa lame est moindre $(7$ à $10 \mathrm{~mm})$ que celle de la scie de travers $(12 \mathrm{~mm})$. Sa denture est triangulaire isocèle et agit dans les deux sens de son mouvement. La scie de charpentier est mancuvrée par deux ouvriers, et la pièce à scier est levée sur des tréteaux. On l'utilise pour DÉBITER (to saw up ; to cut up) les bois courbes et pour exécuter les courbes en travers des fibres du bois.

\section{CONTINENTAL FRAME SAW 4 , CROSSCUT SAW 1 : SCIE À DÉBITER (1676), SCIE À DÉBIT, SCIE DE DÉBIT.}

Scie à monture de bois, de grande dimension, à lame fixe, réservée au sciage droit de débit second à travers les fibres du bois.

La scie à débiter est une scie montée de menuisier, mais elle fait partie d'un outillage commun aux partisans du bois. Sa lame est longue $(80$ à $120 \mathrm{~cm})$, épaisse, large ( 8 à $10 \mathrm{~cm}$ ) et fixe dans le plan de la monture; sa DENTURE est TRIANGULAIRE COUCHÉE (skew teeth).

La scie de débit est une grande scie réservée au sciage grossier à travers les fibres du bois, et on l'utilise pour la coupe transversale de plateaux ou de planches de chantier. Elle peut être manœuvrée par un ou deux hommes.

\section{CONTINENTAL FRAME SAW 5 , RIPSAW 1 : SCIE À TENON, SCIE À TENONS.}

Scie à monture de bois, de dimension moyenne, à lame fixe, réservée au sciage droit de finition dans le sens des fibres du bois.

La scie à tenon est une scie de menuisier de structure identique à celle de la scie à débiter, quoique plus petite (long. de la lame : 65 à $85 \mathrm{~cm}$; larg. : 4,5 à $5,5 \mathrm{~cm}$ ). Sa denture triangulaire couchée est fine, et on utilise cette scie pour le sciage précis de finition dans le sens des fibres, et plus particulièrement pour l'exécution des tenons. La scie à tenon fait aussi partie d'une série d'outils que l'on qualifie d'outils «D'ASSEMBLAGE» (joining tools) parce qu'ils sont consacrés presque exclusivement à cet usage.

\section{CONTINENTAL FRAME SAW 6 : SCIE À ARASER, SCIE À RASER (CAN) (rare), SCIE À ENRASER (1694) (archaïque).}

Scie à monture de bois, de dimension moyenne, à lame fixe, réservée au sciage droit de finition à travers les fibres du bois.

La scie à araser est une scie montée de menuisier de dimension moyenne (50 à $80 \mathrm{~cm}$ ). Sa lame est fixe dans le plan de la monture et possède une denture triangulaire très fine, légèrement couchée, et à pas rapproché, pour répondre aux exigences d'un sciage délicat; sa minceur $(4$ à $6 \mathrm{~mm})$ permet de trancher les fibres du bois, sans les arracher. On l'utilise pour faire des coupes fines à tra- 
vers fibres, pour araser des tenons et découper des onglets. C'est une SCIE D'ASSEMBLAGE (joining saw).

10. CONTINENTAL FRAME SAW 7, GERMAN BOW SAW, RIPSAW 2 : SCIE À REFENDRE 1 (1690). SCIE ALLEMANDE.

Scie à monture de bois, de dimension moyenne, à LAME ORIENTABLE (swivel blade), réservée au sciage droit de débit second dans le sens des fibres du bois.

La scie à refendre, dite allemande, est une scie de menuisier analogue aux précédentes, faisant partie de l'outillage commun aux divers artisans du bois. Elle est d'assez grande dimension $(70$ à $80 \mathrm{~cm})$ et nécessite souvent la présence de deux hommes pour la guider. La lame est longue, assez large $(4,5$ à $5,5 \mathrm{~cm})$ et épaisse (env. $8 \mathrm{~mm}$ ); de plus, elle porte des dents grossières inclinées de telle sorte que l'outil ne mord qu'en descendant. La monture est robuste et dite à champtournant, la lame étant orientable grâce à des TOURILlons (swivel; knob) en bois, dont l'un forme la poignée de la scie. Les bras sont légèrement renflés à cette extrémité et percés d'un œil; les tourillons sont reliés à la lame par l'intermédiaire de CHAPERoNs (spigot) en fer dont l'un des côtés est rivé à la lame, et l'autre fixé par une goupille dans la fente du tourillon. La lame peut ainsi prendre une position perpendiculaire au châssis. On se sert de cette scie toujours verticalement, c'est-à-dire que l'ouvrier imprime un mouvement alternatif à la scie dans un plan presque vertical par rapport à la pièce de bois. La scie à refendre est réservée au sciage grossier du bois du fil. Le menuisier l'utilisait autrefois à l'étape de la préparation du bois, pour déligner les pièces dont il voulait se servir, c'est-à-dire les débarrasser de leurs imperfections latérales (restes d'écorce, parties altérées par un nœud ou par la pourriture) en sciant la pièce dans le sens de la longueur de manière à ce que le bois restant soit parfaitement sain; il utilise aujourd'hui la scie à refendre pour les coupes longitudinales de plateaux et de planches, d'après un tracé de débit. Lorsque des tensions internes existent dans la planche, les pièces débitées de grande longueur se cintrent vers l'extérieur; on dit alors qu'elles «tirent à cœur».

Scie à refendre

a) lame orientable

b) tourillon

c) chaperon

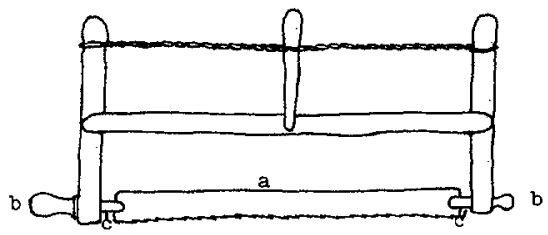

Remarque. Il existe aussi un autre modèle de scie à refendre, de construction antérieure à celui décrit plus haut, la «grande scie à refendre». Elle se présente alors comme une scie de long, mais beaucoup plus petite. La lame est tendue au milieu d'une monture de bois rectangulaire, à mi-chemin entre les montants. Les dimensions de la scie permettent à un homme seul de l'uti- 

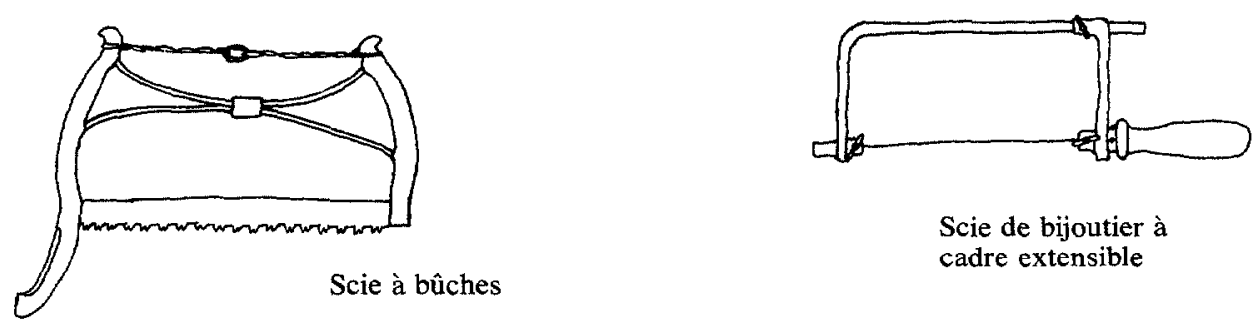

Scie de bijoutier à cadre extensible

liser, en la tenant par les montants. La pièce de bois à refendre est placée sur l'établi et solidement retenue par le valet. Ce modèle était utilisé par les charpentiers, plutôt que par les menuisiers.

\section{CONTINENTAL FRAME SAW 8: SCIE À ARASER DE CÔTÉ, SCIE TOURNANTE (1694).}

Scie à monture de bois, de dimension moyenne, à lame orientable, réservée au sciage de finition, à travers les fibres du bois.

La scie à araser de côté, ou scie tournante, est une scie à araser de dimension moyenne $(55$ à $65 \mathrm{~cm})$, qui présente une lame mobile dans sa monture (larg. $4 \mathrm{~cm}$ ), grâce à des tourillons de bois ou de métal. Elle est utilisée à l'étape de la finition, pour le sciage des ABOUTs (butt) et l'affleurement des tenons : on supprime avec cette scie l'extrémité de battants et de tenons sans être gêné par la monture. C'est une scie d'assemblage.

\section{CONTINENTAL FRAME $S A W 9$ : SCIE À DOUBLE LAME.}

Scie à monture de bois, de dimension moyenne, comportant deux lames, l'une fixe, et l'autre orientable.

La scie à double lame est une scie montée de menuisier très particulière : elle réunit sur la même monture deux lames, l'une de scie à débiter (lame fixe), et l'autre de scie à chantourner (lame mobile). La tension des lames est maintenue par le sommier, qui pousse ou ramène vers lui les bras de la scie, selon qu'on la tourne dans un sens ou dans l'autre.

\section{BOW SAW 2 (1816), TURNING SAW 1 (1846), BRITISH BOW SAW, FRAMED BOW SAW, SWEEP SAW (1846) (archä̈qu) : SCIE À CHAN- TOURNER, SCIE À TOURNER (1676).}

Scie à monture de bois, de dimension moyenne, à lame orientable, réservée au sciage cintré de finition.

La scie à chantourner, ou scie à tourner, est une scie de menuisier de dimension moyenne $(70$ à $80 \mathrm{~cm})$. Elle est de structure identique à celle de la scie à refendre. Sa lame, mobile dans le plan de la monture, doit être aussi étroite que possible ( 5 à $15 \mathrm{~mm}$ ) afin de ne pas gêner la pénétration dans le bois, 
et son épaisseur est souvent renforcée (8 à $12 \mathrm{~mm})$; de plus, la voie doit être large pour que la lame puisse suivre avec précision tous les contours du travail.

La lame de la scie à chantourner est à QUEUE NATURELLE (tang) et est fixée directement dans la fente des tourillons; cependant, à la différence des autres scies montées à lame orientable, la GOUPILLE supérieure DE FIXATron (blade holder pin) n'est jamais cachée dans l'œil du bras, et est facilement accessible afin que l'on puisse démonter la lame pour l'exécution de CHANTOURNEMENTS FERMÉS (interior curves), c'est-à-dire au milieu d'une pièce de bois, plutôt que sur les bords de celle-ci.

La denture est triangulaire et permet à la scie de couper en montant et en descendant.

On se sert de la scie à chantourner comme de la scie à refendre, soit toujours verticalement par rapport à la pièce à couper. C'est une scie d'assemblage, qui sert à découper le bois suivant les courbes du tracé.

Remarque I. Le terme «sweep saw» s'applique, en fait, à toutes les scies montées à lame orientable; cependant, la scie à chantourner étant la seule scie de ce type à être utilisée par les anglophones, «sweep saw» est donc devenu synonyme de «bow saw».

Remarque 2. Dans le modèle anglais de la scie à chantourner, les bras de la monture sont légèrement convexes; de plus, l'assemblage bras-traverse se fait à tenon et à mortaise plutôt qu'à ENFOURCHEMENT (bridle joint); la forme de la monture et l'assemblage sont donc les traits qui distinguent essentiellement la scie montée "British" de celle de type «continental».

Remarque 3. Le terme "turning saw" désigne très souvent une scie que l'on emploie pour le sciage cintré (voir BOW $S A W_{2}$, NARROW OPEN PIT SAW, COMPASS SAW et KEYHOLE SAW).

14. BUCK SAW, BUCKSAW, WOODCUTTER'S SAW (1846), WOOD SAW, FIREWOOD SAW, BILLET SAW (archä̈que) : SCIE À BUCCHES ${ }_{1}$, SCIE À BÛCHES SAVOYARDE.

Scie à monture de bois, de dimension moyenne, à lame fixe, réservée au sciage droit de débit premier à travers les fibres du bois.

La scie à bûches est une scie à monture de grande dimension $(60$ à $90 \mathrm{~cm})$; elle diffère cependant des autres scies montées en ce que l'un des bras est plus long que l'autre, et tient lieu de poignée. Ses dents triangulaires symétriques travaillent dans les deux sens. C'est une scie de travers très robuste qu'on utilise surtout pour le TRONÇONNAGE (logging) et la coupe de bois de chauffage.

\section{COOPER'S HEAD SAW, COOPER'S FRAME SAW : SCIE À CADRE DU TONNELIER, SCIE À CHANTOURNER 2, FEUILLET À TOURNER, GRANDE SCIE DE TONNELIER.}

Scie à monture de bois, de dimension moyenne, à lame fixe, réservée au sciage cintré. 
La grande scie de tonnelier est une scie analogue à la scie à bûches. C'est une scie à monture de dimension moyenne $(\mathrm{env} .90 \mathrm{~cm}$ ) à lame étroite $(1$ à $2 \mathrm{~cm}$ ), dont l'un des bras est plus long que l'autre et tient lieu de poignée. Cependant, à la différence de la scie à bûches, ses dents pointent vers le haut, et ne travaillent donc qu'en descendant.

Il existe plusieurs modèles de cette scie dont certains ne possèdent qu'un bras; la corde de tension, partant de l'unique bras en diagonale est fixée au bout de la traverse qui s'incurve pour recevoir la lame.

Cette scie est utilisée par le tonnelier pour chantourner, c'est-à-dire scier en rond les fonds de barrique d'après le tracé fait au compas.

16. BOW $\mathrm{SAW}_{3}$ : SCIE À ARC, SCIE À ARCHET 1 , SCIOTTE 1 (CAN).

Scie à monture de bois, de dimension moyenne, à lame fixe, réservée au sciage droit de débit premier à travers les fibres du bois.

La scie à archet est une des scies les plus anciennes. On la faisait avec le tronc d'un très jeune arbre (env. $2,5 \mathrm{~cm}$ de diamètre) auquel on donnait la forme d'un arc en fixant à ses extrémités une corde que l'on tordait à l'aide d'un garrot jusqu'à ce que l'arbre ait la forme et la tension désirées; on y fixait alors la lame (env. $90 \mathrm{~cm}$ ). On l'utilisait pour de petits travaux de débit de travers, tel l'ébranchage. C'est la scie à bûches à cadre d'acier tubulaire qui en est la version moderne.

17. TUBULAR BOW SAW (1914), BOW SAW 4, LOG SAW, TUBULAR FRAME-SAW, BUSHMAN'S SAW (archaïque), FORESTER'S SAW (archaïque): SCIE À BÛCHES 3 (MONTURE TUBULAIRE), SCIE À AR$\mathrm{CHET}_{2}$, SCIE À CADRE 2, SCIOTTE 2 (CAN).

Scie à monture métallique, de dimension moyenne, à lame fixe, réservée au sciage droit de débit premier, à travers les fibres du bois.

Cette scie à bûches est la version moderne de la scie à arc. C'est une scie légère, de dimension moyenne $(60$ à $90 \mathrm{~cm})$, dont la lame $(2$ à $3 \mathrm{~cm}$ de large) est maintenue rigide par un CADRE D'ACIER TUBULAIRE (tubular steel bow). On l'utilise pour les petits travaux de débit de travers (tronçonnage de billes, émondage, abattage de petits arbres, etc.)

Remarque 1. "Tubular bow saw " est la forme étoffée; «bow saw est le terme le plus courant.

Remarque 2. «Log saw» est un terme qui s'applique, en fait, à toutes les scies qu'on utilise en région boisée (scie passe-partout, scie à bûches, etc.)

18. FRET SAW (1914), FRETSAW, SCROLL SAW, DEEP THROAT COPING SAW (inusité), BRACKET SAW (archaïque), BUHL SAW (1846) (archaïque), INLAYING SAW (1846) (archaïque) : SCIE À DÉCOUPER (1765), SCIE DE MARQUETERIE (1694), SCIE BOCFIL, BOCFIL (n.f.). 
Scie à monture métallique, de très petite dimension, à lame fixe, réservée au sciage cintré de finition.

La scie à découper, ou scie bocfil, est une scie montée de petite dimension (env. $15 \mathrm{~cm}$ ) qui comporte une lame fixe, et étroite, retenue sous tension par un CADRE MÉTALLIQUE (metal bow; metal bow frame) profond ( 20 à $30 \mathrm{~cm}$ ), en forme de $U$ et muni d'une poignée de bois. Le cadre métallique est suffisamment rigide pour MAINTENIR à lui seul LA TENSION (to hold the tension) de la lame que l'on fixe à la monture à l'aide de deux vis à ailettes. La lame est si étroite (env. $1,5 \mathrm{~mm}$ ) qu'il n'est pas nécessaire qu'elle soit orientable pour suivre la courbe des divers tracés.

Sa DENTURE, triangulaire couchée, est très FINE (fine teeth) et peut comporter jusqu'à 13 dents au $\mathrm{cm}$. Les dents doivent pointer vers la poignée et coupent donc dans le retour de la scie. On utilise la scie à découper pour les travaux délicats de finition dans de minces planches de bois ou des feuilles de placage, p. ex.

Remarque: Le terme «buhl saw» vient de «Buhl» qui est la graphie allemande du nom d'un artisan parisien, A.C. Boulle (1642-1732), dont la spécialité était la marqueterie. "Buhl saw» était l'appellation anglaise la plus courante de la scie à découper jusqu'en 1914; depuis, ce terme est complètement sorti de l'usage, et a été remplacé par «fret saw».

\section{COPING SAW (1920), SCRIBING SAW (inusité: SCIE À CHANTOUR- NER 3.}

Scie à monture métallique, de très petite dimension, à lame orientable, réservée au sciage cintré de finition.

La scie à chantourner est une scie montée de petite dimension $(15$ à $17 \mathrm{~cm})$, de structure semblable à celle de la scie de marqueterie. Elle comporte une lame fixe et étroite ( 2 à $4 \mathrm{~mm}$ ) retenue sous tension par un cadre en métal rigide (11,5 à $16,5 \mathrm{~cm}$ de profond), en forme de $U$ et muni d'une poignée de bois ; des goupilles fixent les extrémités de la lame aux chaperons de métal qui donnent à la lame son orientation. La tension de la lame est ajustée par la poignée que l'on tourne dans un sens ou dans l'autre, pour TENDRE (to tighten) ou DÉTENDRE (to slacken) la monture. Sa denture très fine est triangulaire couchée, et ne coupe donc que dans un sens, mais on peut faire pointer les dents vers la poignée ou à l'opposé, selon le travail à exécuter. On utilise cette scie à chantourner soit pour l'exécution des courbes de très petit diamètre (moulures, p. ex.) que l'on ne peut réaliser avec la scie baïonnette, soit que pour le DÉCOUPAGE (scrollwork) des contreplaqués et des planches minces.

Remarque. La scie à chantourner est plus récente que la scie à découper, et c'est justement le nom français de «scie à découper» qui serait à l'origine du terme anglais "coping saw». 
20. PIERCING SAW (1846), JEWELLER'S PIERCING SAW, LIGHT WEIGHT FRET SAW (E.U.) (inusité) : SCIE DE BIJOUTIER, SCIE À CHANTOURNER (DU BIJOUTIER).

Scie à cadre métallique, de très petite dimension, à lame fixe, réservée au découpage de feuilles de métal.

La scie à chantourner du bijoutier est une scie montée, de très petite dimension $(8$ à $13 \mathrm{~cm})$. De structure semblable à celle de la scie à découper, le CADRE en est cependant moins profond $(7 \mathrm{à} 9 \mathrm{~cm})$ et peut, dans certains modèles EXTENSIBLES (adjustable bow frame; sliding frame) s'adapter à des lames de différentes longueurs; sa lame, très fine, peut avoir jusqu'à 32 dents au cm, et est fixée à la monture à l'aide de vis à ailettes. Le menuisier utilise cette scie pour l'exécution de sciages courbes, dans de minces feuilles de métal (acier, cuivre, bronze) qui servent d'éléments de décoration.

21. HACK SAW, SMITH'S FRAME SAW (1846) (archaïque): SCIE À MÉTAUX, SCIE À FER (CAN).

Scie à monture métallique, de petite dimension, à lame fixe, réservée à la coupe de métal.

La scie à métaux est une scie de petite dimension $(20$ à $30 \mathrm{~cm})$, montée dans un cadre de métal rigide, parfois tubulaire peu profond, muni d'une poignée ouverte, ou droite; le cadre peut, dans certains modèles extensibles, recevoir des lames de différentes longueurs $(20,25$ ou $30 \mathrm{~cm}$, p. ex.) La lame de la scie est percée à chaque extrémité et est fixée à la monture à l'aide de vis ou de rivets, et sa tension est maintenue par un écrou à ailettes. Le menuisier l'utilise pour couper vis, clous et boulons.

Marie ProulX 